\title{
Sepsis and AKI in Clinical Emergency Room Patients: The Role of Urinary NGAL
}

\author{
Hong Si Nga, Pamela Medeiros, Precil Menezes, Ramaiane Bridi, \\ André Balbi, and Daniela Ponce
}

São Paulo State University (UNESP), Distrito de Rubião Junior, s/n, 18618-970 Botucatu, SP, Brazil

Correspondence should be addressed to Daniela Ponce; dponce@fmb.unesp.br

Received 4 February 2015; Revised 19 May 2015; Accepted 21 May 2015

Academic Editor: Anastasia Kotanidou

Copyright (C) 2015 Hong Si Nga et al. This is an open access article distributed under the Creative Commons Attribution License, which permits unrestricted use, distribution, and reproduction in any medium, provided the original work is properly cited.

\begin{abstract}
Background. Few studies have investigated the predictive properties of urinary (u) NGAL as an AKI marker in septic population. Objectives. This study evaluated the efficacy of uNGAL as predictor of AKI and death in septic patients admitted to the clinical emergency room (ER). Methodology. We prospectively studied patients with sepsis admitted to the ER. Urine was analyzed for NGAL within the first 24 hours after admission (classified as NGAL1), between 24 and $48 \mathrm{~h}$ (NGAL2), and at moment of AKI diagnosis (NGAL3). Results. Among 168 septic patients admitted to ER, 72\% developed AKI. The uNGAL and its relationship with creatinine $(\mathrm{Cr})$ were high in septic patients but statistically higher in those with sepsis and AKI. The uNGAL1 and uNGAL2, as well as $\mathrm{uNGAL} 1 / \mathrm{uCr} 1$ and $\mathrm{uNGAL} 2 / \mathrm{uCr} 2$, were good predictors for AKI (AUC-ROC 0.73, 0.70, 0.77, and 0.84, resp.). The uNGAL1 and uNGAL1/uCr1 were poor predictors for death (AUC-ROC 0.66 and 0.68, resp.), whereas uNGAL2 and uNGAL2/uCr2 were better predictors (AUC-ROC 0.70 and 0.81 , resp.). Conclusion. The uNGAL is highly sensitive but nonspecific predictor of AKI and death in septic patients admitted into ER.
\end{abstract}

\section{Introduction}

Sepsis is defined as systemic inflammatory response syndrome associated with infection. It is a primary cause of morbidity and mortality in patients admitted to emergency clinical room (ER) and in intensive care units (ICU) [1-3]. It is a well-known risk factor for the development of acute kidney injury (AKI), occurring in approximately $51 \%$ of patients with septic shock [4-6]. The presence of AKI leads to significant impact on morbidity, increased length of stay in hospital, and high costs, and it is an independent risk factor for mortality $[4,7,8]$.

Neutrophil gelatinase-associated lipocalin (NGAL) is a rising biomarker for early diagnosis of AKI in different scenarios. NGAL levels in both plasma (p) and urine (u) increase soon after the renal insult and they seem to detect AKI hours or days before creatinine ( $\mathrm{Cr}$ ) [9-12]. Although considered an early biomarker, NGAL levels can be elevated after activation of neutrophils, suggesting influence of systemic inflammation and infections [13-16].
Few studies have investigated the predictive properties of NGAL as an AKI marker in a septic population. Studies on pediatric ICU patients have shown pNGAL to be a nonspecific predictor [17] and UNGAL to be a good predictor of AKI [18]. In these two pediatric studies AKI and sepsis coincided to a great extent. This is common in ICU patients and might obstruct the interpretation of elevated NGAL in plasma and urine. Indeed, Bagshaw et al. [19] in a study that included 83 AKI patients showed that both p- and uNGAL were higher in septic versus nonseptic patients. Mårtensson et al. [20] performed a study that evaluated 65 septic patients admitted to ICU and showed that pNGAL was not a good predictor of AKI because it was elevated in septic patients without AKI, probably due to the systemic infections.

Given the higher mortality rate of patients with sepsis and AKI and lack of studies in ER, we decided to investigate the role of uNGAL as predictor of AKI and death in septic patients admitted to ER.We believe uNGAL is predictor of AKI and death in septic patients admitted to ER. 


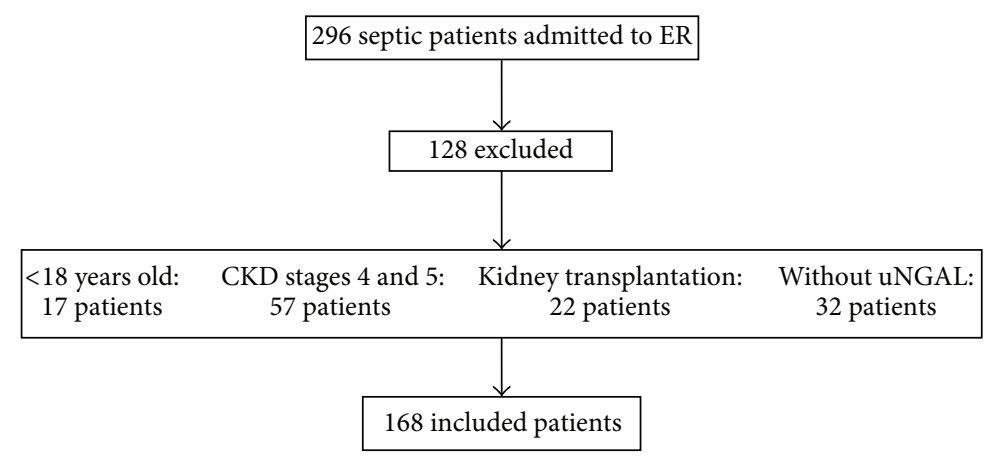

FIGURE 1: Screening and enrollment.

\section{Materials and Methods}

2.1. Study Population. We screened all septic patients admitted through the internal medicine department to the ER of our University Hospital from January 2013 to May 2014 for enrollment in a prospective cohort study designed to study the development of AKI following sepsis. We included patients 18 years of age or older who had sepsis according to "Survival Sepsis Campaign 2012" [21] and exclusion criteria were patients with chronic kidney disease stages 4 and 5 (creatinine clearance lower than $30 \mathrm{~mL} / \mathrm{min} / 1.73 \mathrm{~m}^{2}$ ) estimated by the modification of diet in renal disease (MDRD) equation [22] and patients undergoing kidney transplantation. Complete data on inclusions and exclusions are shown in Figure 1.

The Ethics Committee of the Botucatu School of Medicine, UNESP, approved this study with a waiver of informed consent given its observational nature.

AKI was defined and classified according to AKIN criteria $[23,24]$. Baseline Cr was defined as the lowest Cr value in the last 6 months before AKI or, for those without this measurement, the lowest value achieved during hospitalization in the absence of dialysis $[25,26]$.

AKI was considered to have occurred on the first day that any criterion was met, though full staging continued through discharge from hospital or death, whichever came first. Day 0 was defined as the calendar day of ER, and thus its length varied depending on time of presentation. We determined vital status at the time of discharge from the hospital for all patients.

Our 5-day study time frame was designed to capture most cases of early AKI and to reduce the confounding effects of time-varying interventions (e.g., nephrotoxic medications) and late complications (e.g., sepsis related to mechanic ventilation (MV)) which would likely have had a greater impact in cases of AKI occurring later in the hospital course.

2.2. Biochemical Analysis. Samples of blood were collected once daily during 5 days or earlier if discharged from hospital or death. Urine was analyzed for NGAL and Cr within the first 24 hours after admission (classified as NGAL1), between 24 and $48 \mathrm{~h}$ (NGAL2), and at moment of AKI diagnosis (NGAL3). The samples were centrifuged and stored at minus 80-degree Celsius and were analyzed subsequently. NGAL was measured by the enzyme linked immunosorbent assay (ELISA).

Expected normal uNGAL level was less than $0.2 \mathrm{ng} / \mathrm{mL}$. We performed dosage of uNGAL in 20 healthy subjects between 30 and 50 years old and the mean was $0.2 \pm$ $0.029 \mathrm{ng} / \mathrm{mL}$.

2.3. Statistical Analysis. Data analysis was performed using SAS for Windows (version 9.2, SAS Institute, Cary, NC, USA, 2012). Continuous variables with normal distribution were described using means \pm standard deviation and those with a nonnormal distribution as median and interquartile range. Categorical variables were presented as $n(\%)$. For the analysis of continuous variables, Student's $t$-test was used for data with a parametric distribution and the Kruskal-Wallis test for nonnormal data. For the analysis of categorical variables a chi-square test was used.

Diagnostic characteristics of UNGAL in predicting AKI and death were assessed by calculation of the area under the receiver operating characteristic curve (AUC-ROC). AUCROC analysis was performed by comparing AKI patients with all non-AKI patients and by comparing survivor patients with those nonsurvivor patients. In all tests, differences were considered significant at $5 \%$.

\section{Results}

One hundred sixty-eight patients were included in the final analysis (Figure 1). Mean age was $68.0 \pm 15.4$ years, $57.7 \%$ were male, most of them had comorbidities (65.4\%), and hypertension, cardiovascular disease, and diabetes mellitus were the most frequent (in 50.6, 29.7, and $26.1 \%$ of patients, resp.). APACHE II score was $19.70 \pm 7.1$ and the need for mechanical ventilation and noradrenalin use in the first 24 hours after admission to ER was 21.4 and $55.4 \%$, respectively. Septic shock was the primary sort of septic state (55.4\%). The main source of infection was the lung (48.8\%), followed by the urinary tract (18\%). The pathogen was isolated in culture in only $38 \%$ of cases. Patients were mainly directed towards the ICU (51.8\%), while only 53 patients $(31.5 \%)$ were referred to the ward and the others stayed at the ER. Within the first 
TABle 1: Patients demographics and clinical characteristics $(n=$ 168).

\begin{tabular}{|c|c|}
\hline Characteristics & Septic patients $(N=168)$ \\
\hline Male sex $n(\%)$ & $97(57.7)$ \\
\hline Age (years) & $68 \pm 15.4$ \\
\hline MBP & $73.3 \pm 23$ \\
\hline \multicolumn{2}{|l|}{ Comorbidities $n(\%)$} \\
\hline Hypertension & $85(50.6)$ \\
\hline Diabetes & $44(26.1)$ \\
\hline Dyslipidemia & $18(10.7)$ \\
\hline Cardiovascular disease & $50(29.7)$ \\
\hline Liver disease & $6(3.5)$ \\
\hline CKD & $12(7.1)$ \\
\hline Baseline creatinine & $0.82 \pm 0.3$ \\
\hline Noradrenaline use $n(\%)$ & $93(55.3)$ \\
\hline \multicolumn{2}{|l|}{ Classification of sepsis $n(\%)$ : } \\
\hline Sepsis & $26(15.4)$ \\
\hline Severe sepsis & $49(29.1)$ \\
\hline Septic shock & $93(55.3)$ \\
\hline \multicolumn{2}{|l|}{ Source of infection $n(\%)$ : } \\
\hline Urine & $30(18)$ \\
\hline Lung & $81(48.8)$ \\
\hline Mechanical ventilation $n(\%)$ & $36(21.4)$ \\
\hline Blood transfusion $n(\%)$ & $13(7.7)$ \\
\hline Steroids use $n(\%)$ & $29(17.2)$ \\
\hline \multicolumn{2}{|c|}{ Time for antibiotics administration } \\
\hline$<1 \mathrm{~h} n(\%)$ & $83(50.92)$ \\
\hline$>1 \mathrm{~h} n(\%)$ & $80(49.08)$ \\
\hline AKI $n(\%)$ & $121(72.02)$ \\
\hline At admission & $87(71.9)$ \\
\hline During hospitalization & $34(28.1)$ \\
\hline Urine output in $24 \mathrm{~h}(\mathrm{~mL})$ & $900(450-1425)$ \\
\hline Urine output (mL/kg/h) & $0.70(0.4-1.05)$ \\
\hline APACHE II & $19.67 \pm 7.11$ \\
\hline Dialysis n (\%) & $15(8.93)$ \\
\hline \multicolumn{2}{|l|}{ Outcome $n(\%)$} \\
\hline Discharge & $92(55.7)$ \\
\hline Death & $73(44.2)$ \\
\hline
\end{tabular}

Values expressed as mean and standard deviation or median and interquartile range.

AKI: acute kidney injury, MBP: mean blood pressure, CKD: chronic kidney disease, ATN-ISS: acute tubular necrosis individual severity score, and ICU: intensive care unit.

five ER days, 121 subjects (72\%) developed AKI. The mortality rate was $44 \%$ (Table 1 ).

Among AKI patients, 87 (71.9\%) already had the diagnosis on admission to the ER, while 34 of them developed AKI during hospitalization. Most of patients were classified as AKIN 3 (43\%), while AKIN 1 occurred in 35 patients (28.9\%) and AKIN 2 in 34 (28.1\%).

uNGAL1 and UNGAL2 in AKI group showed higher values than non-AKI group: 3.86 (2.6 to 9.5) versus 3.5 (0.85); $p=0.003$ and $3.03(0.65-4.33)$ versus $2.76 \mathrm{ng} / \mathrm{mL}(2.3-$ 7.83); $p=0.009$, respectively, and $\mathrm{uNGAL} / \mathrm{uCr}$ in the first

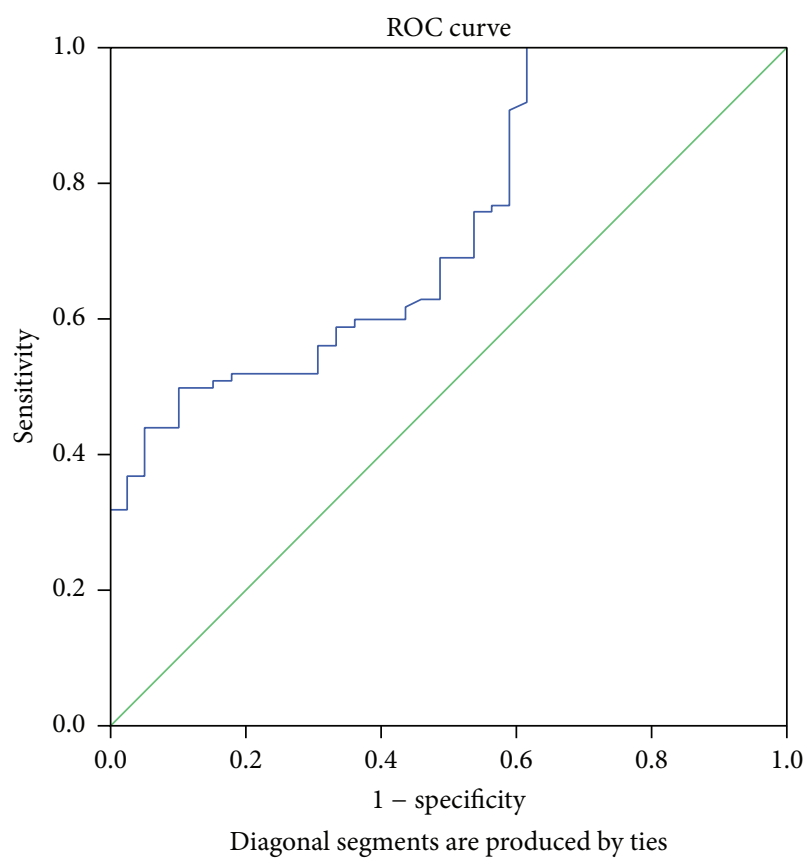

FIGURE 2: ROC analysis of UNGAL measure on day 1 of admission to the ER in septic patients with AKI versus without AKI.

24 h: $75.08(37-165) \times 53.31$ (17.79 to 102.2); $p<0.0001$, and between 24 and $48 \mathrm{~h}$ after admission: $77.2(29.4$ to 160.6$) \times$ 60.29 (17.56 to 85.64$) ; p=0.02$ (Table 2).

uNGAL1 was higher in nonsurvival group when compared with survival patients $(4.88(2.19-9.51) \times 3.30 \mathrm{ng} / \mathrm{mL}$ (1.76-6.18), $p=0.01)$. The two groups were similar in uNGAL2 $(p=0.16)$, as well as uNGAL/uCr in the first $24 \mathrm{~h}(p=0.72)$ and between 24 and $48 \mathrm{~h}$ after admission $(p=0.63)$ (Table 3$)$.

Figures 2-5 display the receiver operator curves (ROC) for UNGAL as predictor of AKI. The areas under the curve for uNGAL1, uNGAL2, uNGAL1/uCr1, and uNGAL2/uCr2 were $0.73,0.70,0.77$, and 0.84 , respectively. Both uNGAL and $\mathrm{uNGAL} / \mathrm{uCr}$ were good predictors of AKI within the next $48 \mathrm{~h}$. The optimal cutoff value of each one of them had sensitivity and specificity of 0.63 and $0.46,0.63$ and $0.44,0.7$ and 0.38 , and 0.75 and 0.43 , respectively (Table 4 ).

Figure 6 shows the values of uNGAL at different moments $(1,2$, and 3$)$ in the group of septic patients that developed AKI during the hospitalization. The expression of uNGAL seemed to follow a bimodal pattern around the development of AKI with an early peak preceding AKI followed by a second peak after AKI was established, which was observed only in patients with no AKI at admission.

Subanalysis was performed involving only patients who did not present AKI at admission to ER $(n=81)$ and results were better than those described in the general population ( $n=168$ patients). The areas under the curve for uNGAL1, uNGAL2, uNGAL1/uCr1, and uNGAL2/uCr2 were $0.83,0.81$, 0.87 , and 0.89 , respectively. Both uNGAL and uNGAL/uCr were excellent predictors of AKI within the next $48 \mathrm{~h}$. The optimal cutoff value of each one of them had sensitivity and 
TABLE 2: Urinary NGAL values according to presence of acute kidney injury.

\begin{tabular}{|c|c|c|c|}
\hline & $\begin{array}{c}\text { AKI } \\
N=121\end{array}$ & $\begin{array}{c}\text { Non-AKI } \\
N=47\end{array}$ & $p$ \\
\hline \multicolumn{4}{|l|}{ uNGAL (ng/mL): } \\
\hline At moment $1\left(<24 \mathrm{~h}^{*}\right)$ & $3.86(2.6-9.54)$ & $3.56(0.82-5.2)$ & 0.003 \\
\hline At moment $2\left(24-48 \mathrm{~h}^{*}\right)$ & $3.03(0.65-4.33)$ & $2.76(2.3-7.83)$ & 0.009 \\
\hline \multicolumn{4}{|l|}{ uNGAL/uCr (ng/mg): } \\
\hline At moment $1\left(<24 \mathrm{~h}^{*}\right)$ & $75.08(37-165)$ & $53.31(17.79-102.2)$ & $<0.0001$ \\
\hline At moment $2\left(24-48 \mathrm{~h}^{*}\right)$ & $77.2(29.49-160.6)$ & $60.29(17.56-85.64)$ & 0.002 \\
\hline
\end{tabular}

Values expressed as median and interquartile range.

u: urinary; AKI: acute kidney injury; ${ }^{*}$ after admission to emergency room.

TABLE 3: Urinary NGAL values according to patient outcome.

\begin{tabular}{lccc}
\hline & Survivors & Nonsurvivors & $N=74$ \\
& $N=94$ & & \\
\hline uNGAL $(\mathrm{ng} / \mathrm{mL}):$ & & $4.88(2.19-9.51)$ & 0.01 \\
At moment $1\left(<24 \mathrm{~h}^{*}\right)$ & $3.30(1.76-6.18)$ & $3.93(1.89-7.19)$ & 0.16 \\
At moment 2 $\left(24-48 \mathrm{~h}^{*}\right)$ & $2.18(0.89-6.36)$ & $12.42(2.63-19.02)$ & 0.15 \\
At moment 3 (AKI diagnosis) & $6.60(1.66-6.9)$ & $75.08(40-154)$ \\
uNGAL/uCr $(\mathrm{ng} / \mathrm{mg}):$ & & $93.35(57.45-115.81)$ & 0.82 \\
At moment $1\left(<24 \mathrm{~h}^{*}\right)$ & $56.91(27.55-113.92)$ & $263.6(99.28-984.15)$ \\
At moment 2 $\left(24-48 \mathrm{~h}^{*}\right)$ & $46.30(20.81-137.49)$ & 0.63 \\
At moment 3 (AKI diagnosis) & $134.41(35.99-259.43)$ & 0.056 \\
\hline
\end{tabular}

Values expressed as median and interquartile range.

u: urinary; AKI: acute kidney injury; ${ }^{*}$ after admission to emergency room.

specificity of 0.77 and $0.66,0.75$ and $0.78,0.81$ and 0.68 , and 0.79 and 0.67 , respectively (Table 5 ).

Concerning uNGAL as predictor of death, the areas under the curve for uNGAL1, uNGAL2, uNGAL1/uCr1, and uNGAL2/uCr2 were $0.66,0.70,0.68$, and 0.81 , respectively (Figure 7). Only uNGAL2 and uNGAL2/uCr2 were good predictors of septic patients death. The optimal cutoff value of each one of them had sensibility and specificity of 0.88 and $0.54,0.95$ and $0.59,0.71$ and 0.45 , and 0.71 and 0.48 , respectively (Table 6).

\section{Discussion}

This is the first study of septic adult patients admitted to ER to undergo prospective evaluation of UNGAL as a biomarker for AKI and death. Among 168 patients with sepsis and septic shock admitted into ER, 121 (72\%) developed AKI defined by AKIN classification [24] and mortality rate was $44 \%$. There are few studies on AKI in ER and these findings are consistent with the previous studies performed in ICU.

Many studies have reported that AKI is more frequently observed in patients with sepsis and septic shock than in patients with other conditions $[23,24,27,28]$. An observational cohort study of 390 patients with septic shock in a single center ICU for about 2 years reported nearly 2 out of 3 patients experiencing AKI. In a recent retrospective multicenter study of 4532 patients with septic shock, a similar percentage of patients (64.4\%) developed AKI [27]. Challiner et al. [28] performed a retrospective study with 745 patients admitted to the emergency department and evaluated the presence or absence of AKI according to AKIN criteria. AKI incidence was $25.4 \%$ overall, with approximately onethird present on admission and two-thirds developing after admission.

Herein we show that uNGAL and its relation to $\mathrm{uCr}$ were significantly increased within the first 48 hours of admission to the ER in septic AKI patients compared to healthy controls and septic patients without AKI. In addition, uNGAL on day 1 of admission to ER was significantly increased in nonsurvivors septic patients compared to survivors ones. uNGAL therefore appears to be a highly sensitive predictor of AKI and death in this population.

NGAL is a protein with a molecular weight of $25 \mathrm{kDa}$ expressed at low concentrations in different tissues and upregulated especially in injured epithelial cells. Because of that, pNGAL concentration can be high in septic patients, even in the absence of AKI. Then, it may be considered a marker of sepsis, as well as an early biomarker of AKI [29], as shown in several clinical studies [9, 10, 18, 30-32].

In study of Dellinger et al. [21], pNGAL and uNGAL were evaluated as predictors of AKI, but the ability of pNGAL to predict AKI in patients with septic shock was poor with an AUC-ROC (0.67) compared to the ability of uNGAL with an AUC-ROC (0.86). The UNGAL was a better predictor of 
TABLE 4: Urinary NGAL sensitivity and specificity in general septic patients $(n=168)$.

\begin{tabular}{lcccccc}
\hline & AUC-ROC & $p$ & Cutoff & Sensitivity & Specificity & CI (95\%) \\
\hline uNGAL1 & 0.73 & 0.04 & 3.36 & 0.63 & 0.46 & $(0.64-0.82)$ \\
uNGAL2 & 0.70 & 0.01 & 2.73 & 0.63 & 0.70 & $(0.55-0.85)$ \\
uNGAL/uCr1 & 0.77 & 0.04 & 54.8 & 0.75 & 0.38 & $(0.68-0.85)$ \\
uNGAL/uCr2 & 0.84 & 0.001 & 46.4 & 0.43 & $(0.73-0.94)$ \\
\hline
\end{tabular}

AUC-ROC: receiver operating characteristic curve; Cr: creatinine.

TABLE 5: Urinary NGAL sensitivity and specificity in septic patients without AKI at admission $(n=81)$.

\begin{tabular}{lccccrr}
\hline & AUC-ROC & $p$ & Cutoff & Sensitivity & Specificity & CI (95\%) \\
\hline uNGAL1 & 0.83 & 0.03 & 3.16 & 0.77 & 0.66 & $(0.64-0.81)$ \\
uNGAL2 & 0.81 & 0.01 & 3.83 & 0.75 & 0.78 & $(0.52-0.79)$ \\
uNGAL/uCr1 & 0.87 & 0.02 & 53.8 & 0.81 & 0.68 & $(0.58-0.78)$ \\
uNGAL/uCr2 & 0.89 & 0.0001 & 47.4 & 0.87 & 0.67 & $(0.64-0.71)$ \\
\hline
\end{tabular}

AUC-ROC: receiver operating characteristic curve; Cr: creatinine.

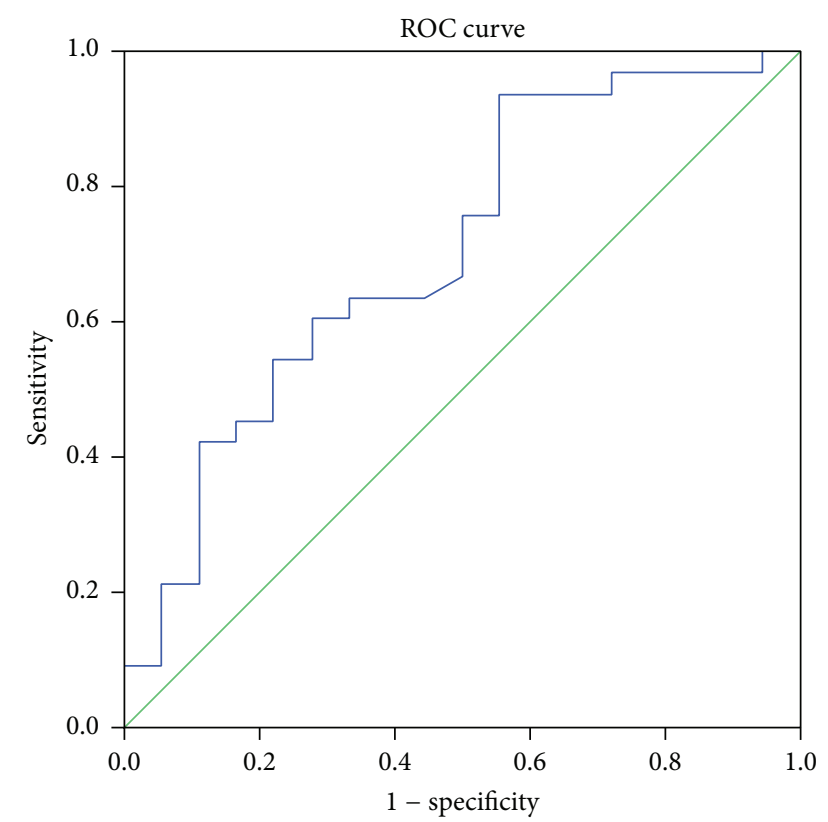

Diagonal segments are produced by ties

Figure 3: ROC analysis of uNGAL measure on day 2 of admission to the ER in septic patients with AKI versus without AKI.

AKI in septic patients than pNGAL probably because it was less affected by presence of sepsis. The pNGAL can be high because of its release into the bloodstream by the systemic activation of neutrophils due to sepsis. The physiological function of the uNGAL is unknown; however, it has a role in renal morphogenesis [33]. The proteomic analysis of studies using animal models revealed UNGAL protein as the earliest product after kidney insult [31], representing better the kidney damage than the pNGAL.

Similar results were found in pediatric patients [17] also with septic shock in ICU and the AUC-ROC (0.67), shown to be more sensitive predictor than specific. As the proper sepsis activates and increases the release of NGAL from neutrophils,

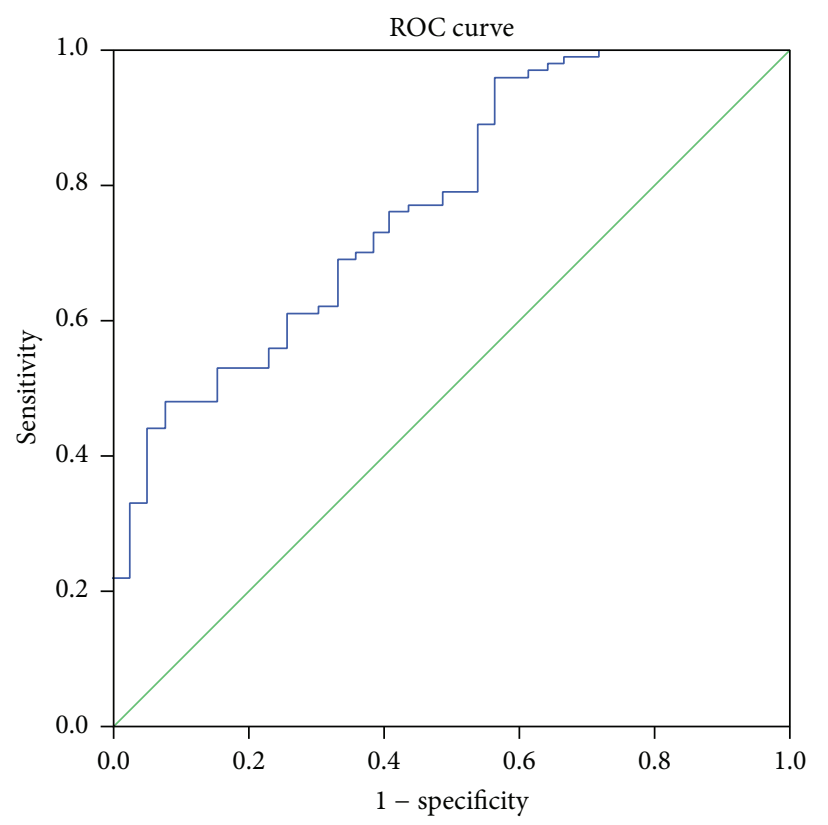

FIGURE 4: ROC analysis of uNGAL/uCr measure on day 1 of admission to the ER in septic patients with AKI versus without AKI.

it is questionable whether it can impair the ability to predict AKI.

In the current study, uNGAL in healthy adults was much lower (median $0.2 \mathrm{ng} / \mathrm{mL}$, IQR $0-1.1 \mathrm{ng} / \mathrm{mL}$ ) than that reported in other studies [18-20]. These differences are likely related to the different techniques used to measure NGAL in different studies.

In our study both $\mathrm{UNGAL}$ and its relation to $\mathrm{uCr}$ on day 1 and day 2 after admission of septic patients to the ER were good predictors of AKI. In the current study, ROC analysis suggested that uNGAL2/uCr2 had an excellent accuracy (0.84) and a high sensitivity for predicting AKI (75\%), albeit with relatively poor specificity $(46 \%)$ predictor of AKI in septic patients admitted to ER. In subanalysis that involved 
TABLE 6: Urinary NGAL sensitivity and specificity in nonsurvival septic patients.

\begin{tabular}{lcccccc}
\hline & AUC-ROC & $p$ & Cutoff & Sensitivity & Specificity & CI (95\%) \\
\hline uNGAL1 & 0.66 & 0.048 & 2.07 & 0.88 & 0.54 & $(0.51-0.81)$ \\
uNGAL2 & 0.70 & 0.01 & 1.84 & 0.95 & 0.71 & 0.59 \\
uNGAL/uCr1 & 0.68 & 0.02 & 55.9 & $0.56-0.85)$ \\
uNGAL/uCr2 & 0.81 & 0.001 & 69.6 & 0.71 & 0.45 & $(0.54-0.83)$ \\
\hline
\end{tabular}

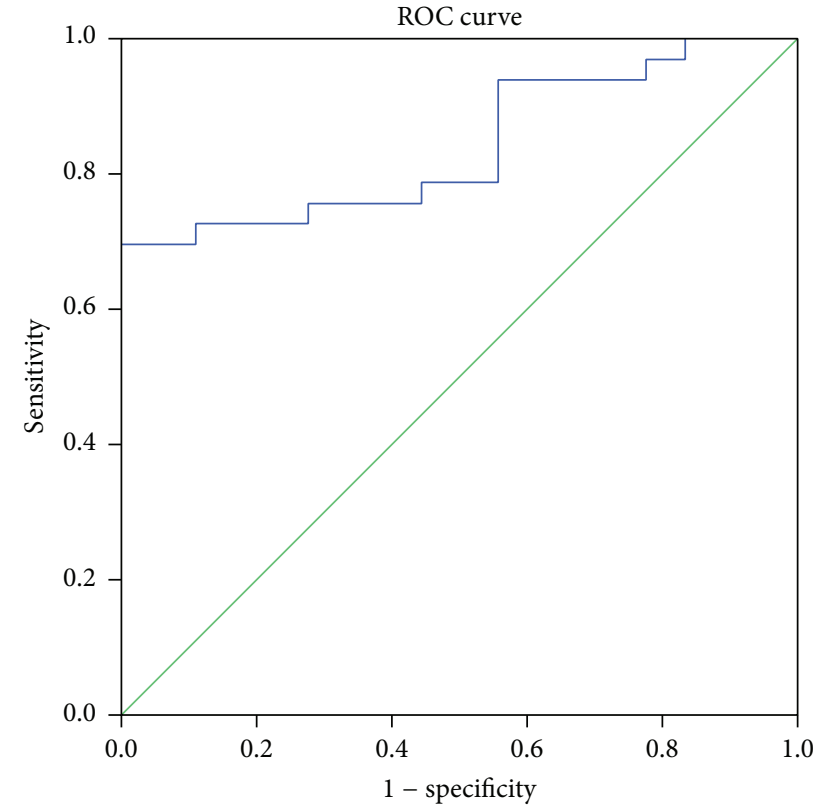

FIGURE 5: ROC analysis of uNGAL/uCr measure on day 2 of admission to the ER in septic patients with AKI versus without AKI.

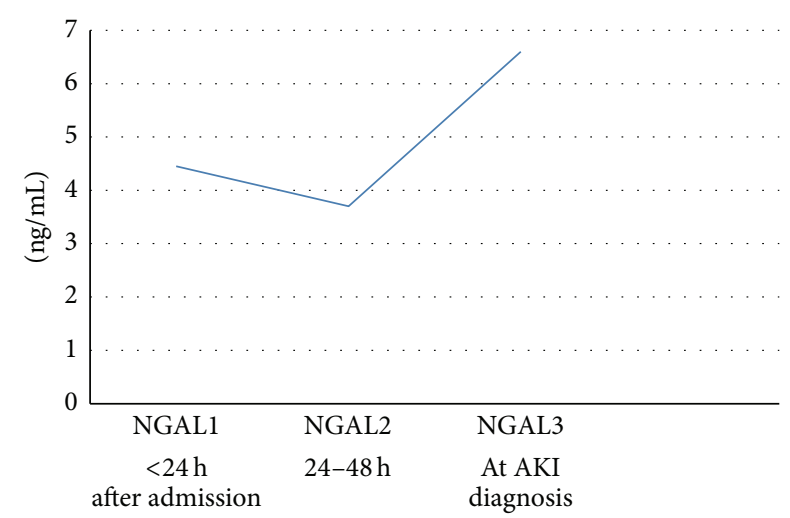

FIgURE 6: Urinary NGAL values at three moments after admission in septic patients undergoing AKI during hospitalization.

only patients who did not present AKI at admission to ER ( $n=81$ ), the results were better than those described in the general population. ROC analysis showed that UNGAL and NGAL/uCr were excellent predictors of AKI within the next $48 \mathrm{~h}(>0.8)$, with a high sensitivity $(>75 \%)$ and a good specificity $(>65 \%)$.

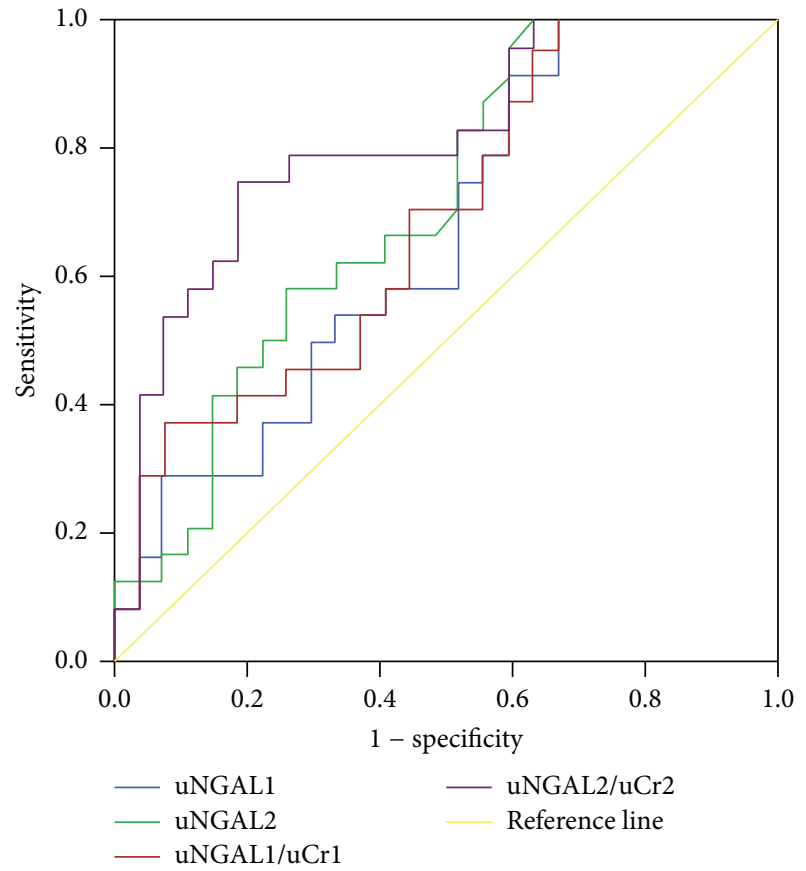

FIGURE 7: ROC analysis of uNGAL and uNGAL/uCr measured on days 1 and 2 of the admission to ER in survivors versus nonsurvivors septic patients.

Our results are similar to the AUC-ROC found in pediatric septic patients by Wheeler et al. [17] and in adults septic patients in study performed by Mårtensson et al. [20]. The authors showed that the AUC was 0.677 (95\% CI 0.557 , $0.786 ; p=0.008$ ) with an optimal cutoff value of $139 \mathrm{ng} / \mathrm{mL}$ (sensitivity $=86 \%$ and specificity $=39 \%$ ).

However, in our study, uNGAL was measured within the first 48 hours of admission to the ER, which is not necessarily the first 48 hours of their disease process. In fact, the vast majority of these patients already had the sepsis diagnosis on admission to the ER. We would therefore expect the UNGAL concentrations to be much higher in patients with septic shock and evolving kidney injury.

In a study that followed children undergoing cardiopulmonary bypass and analyzed UNGAL and pNGAL as predictors of AKI, the concentration of UNGAL greater than $50 \mu \mathrm{g} / \mathrm{L}$ predicted AKI at two hours following procedure in this population, with $100 \%$ sensibility and $98 \%$ specificity, while pNGAL concentrations greater than $25 \mu \mathrm{g} / \mathrm{L}$ did not show such good results, with $70 \%$ sensibility and $94 \%$ specificity [9]. 
We also analyzed the values of UNGAL at different moments ( 1 and 2 ) in the 34 patients that developed AKI during the hospitalization. The expression of UNGAL seemed to follow a bimodal pattern around the development of AKI with an early peak preceding AKI followed by a second peak after AKI was established. Similar results were found by Mårtensson et al. [20]. The first peak was attributed to the excretion of NGAL from neutrophils sequestered in renal tubule and the second peak represented the expression of NGAL released by the tubular cells themselves. Cai et al. [34] studied patients who underwent cardiac surgery and found different molecular forms of uNGAL measured by ELISA at different time points.

We did not find the same for the group that already had AKI by admission; the explanation for that is the fact that the values of NGAL by admission represent the second peak and probably the biomarker should be elevated hours or even days before the hospitalization.

Concerning uNGAL as predictor of death, in our study uNGALl and uNGAL1/uCr1 were poor predictors (AUCROC was 0.7 ). The uNGAL2 and the uNGAL2/uCr2 were good predictor of death in septic patients. ROC analysis suggested that uNGAL2 had a good accuracy (0.7) and high sensitivity for predicting death (95\%), whereas uNGAL2/uCr2 was better, with an excellent accuracy (0.81) and sensitivity for predicting death $(71 \%)$, albeit nonspecific predictor of death (48\%) in septic patients admitted to ER. We believe that adding any other marker, KIM-1, for example, with higher specificity, would help to improve the predictive value of the studied markers.

We speculate that UNGAL2 was better predictor of death in septic patients than UNGAL1 because it may reflect the patient's response to initial treatment of sepsis. If after 24 hours of initial treatment the NGAL u 2 and the uNGAL2/uCr2 remain high, they can predict death of septic patients admitted to ER.

Few studies have shown an association between NGAL and mortality. Nickolas et al. [35] showed that uNGAL was associated with clinical outcomes, including consultation with nephrologist, dialysis, and ICU admission $(\mathrm{OR}=24.71$ (CI: 7.69 to 79.42)). Collins et al. [36] evaluated 399 patients with acute cardiac dysfunction and found that uNGAL between 12 and $24 \mathrm{~h}$ after treatment initiation was predictive of 30 -day mortality $(p=0.02)$.

The present study has some important limitations. It included a small number of patients and was performed in single center. Due to the small number of patients, no analysis of uNGAL according to the stage of AKI or classification of sepsis was performed. The role of UNGAL as a predictor of dialysis also was not evaluated. Despite these limitations, the results of this study allow us to conclude that UNGAL is elevated in septic patients but statistically higher in those with sepsis and AKI and reaffirm the role of UNGAL to predict AKI and death. uNGAL/uCr values on day 2 after admission to ER were the best predictors of AKI and death in septic patients, with being highly sensitive, but nonspecific. We speculate that UNGAL values may be confounded by hydration status and urine output and may therefore need standardization by expressing as a ratio with $\mathrm{uCr}$.
The UNGAL is a highly sensitive but nonspecific predictor of AKI and death in septic patients admitted into ER and further validation of UNGAL as a biomarker of AKI in this population is warranted.

\section{Conflict of Interests}

The authors declare that there is no conflict of interests regarding the publication of this paper.

\section{References}

[1] V. D. Mayr, M. W. Dünser, V. Greil et al., "Causes of death and determinants of outcome in critically ill patients," Critical Care, vol. 10, no. 6, article R154, 2006.

[2] D. C. Angus, W. T. Linde-Zwirble, J. Lidicker, G. Clermont, J. Carcillo, and M. R. Pinsky, "Epidemiology of severe sepsis in the United States: analysis of incidence, outcome, and associated costs of care," Critical Care Medicine, vol. 29, no. 7, pp. 1303-1310, 2001.

[3] E. Silva, M. D. A. Pedro, A. C. B. Sogayar et al., "Brazilian sepsis epidemiological study (BASES study)," Critical Care, vol. 8, no. 4, pp. R251-R260, 2004.

[4] P. Lentini, M. de Cal, A. Clementi, A. D’Angelo, and C. Ronco, "Sepsis and AKI in ICU patients: the role of plasma biomarkers," Critical Care Research and Practice, vol. 2012, Article ID 856401, 5 pages, 2012.

[5] A. Zarjou and A. Agarwal, "Sepsis and acute kidney injury," Journal of the American Society of Nephrology, vol. 22, no. 6, pp. 999-1006, 2011.

[6] C. Alberti, C. Brun-Buisson, H. Burchardi et al., "Epidemiology of sepsis and infection in ICU patients from an international multicentre cohort study," Intensive Care Medicine, vol. 28, no. 2, pp. 108-121, 2002.

[7] K. Singbartl and J. A. Kellum, "AKI in the ICU: definition, epidemiology, risk stratification, and outcomes," Kidney International, vol. 81, no. 9, pp. 819-825, 2012.

[8] J. Chvojka, R. Sýkora, T. Karvunidis et al., "New developments in septic acute kidney injury," Physiological Research, vol. 59, no. 6, pp. 859-869, 2010.

[9] J. Mishra, C. Dent, R. Tarabishi et al., "Neutrophil gelatinaseassociated lipocalin (NGAL) as a biomarker for acute renal injury after cardiac surgery," The Lancet, vol. 365, no. 9466, pp. 1231-1238, 2005.

[10] G. Wagener, M. Jan, M. Kim et al., "Association between increases in urinary neutrophil gelatinase-associated lipocalin and acute renal dysfunction after adult cardiac surgery," Anesthesiology, vol. 105, no. 3, pp. 485-491, 2006.

[11] R. Hirsch, C. Dent, H. Pfriem et al., "NGAL is an early predictive biomarker of contrast-induced nephropathy in children," Pediatric Nephrology, vol. 22, no. 12, pp. 2089-2095, 2007.

[12] H. Bachorzewska-Gajewska, J. Malyszko, E. Sitniewska et al., "Could neutrophil-gelatinase-associated lipocalin and cystatin C predict the development of contrast-induced nephropathy after percutaneous coronary interventions in patients with stable angina and normal serum creatinine values?" Kidney and Blood Pressure Research, vol. 30, no. 6, pp. 408-415, 2007.

[13] S. Y. Xu, K. Pauksen, and P. Venge, "Serum measurements of human neutrophil lipocalin (HNL) discriminate between acute bacterial and viral infections," Scandinavian Journal of Clinical and Laboratory Investigation, vol. 55, no. 2, pp. 125-131, 1995. 
[14] G. Fjaertoft, T. Foucard, S. Xu, and P. Venge, "Human neutrophil lipocalin (HNL) as a diagnostic tool in children with acute infections: a study of the kinetics," Acta Paediatrica, vol. 94, no. 6, pp. 661-666, 1992.

[15] K. Mori, H. T. Lee, D. Rapoport et al., "Endocytic delivery of lipocalin-siderophore-iron complex rescues the kidney from ischemia-reperfusion injury," The Journal of Clinical Investigation, vol. 115, no. 3, pp. 610-621, 2005.

[16] P. Devarajan, "NGAL for the detection of acute kidney injury in the emergency room," Biomarkers in Medicine, vol. 8, no. 2, pp. 217-219, 2014.

[17] D. S. Wheeler, P. Devarajan, Q. Ma et al., "Serum neutrophil gelatinase-associated lipocalin (NGAL) as a marker of acute kidney injury in critically ill children with septic shock," Critical Care Medicine, vol. 36, no. 4, pp. 1297-1303, 2008.

[18] M. Zappitelli, K. K. Washburn, A. A. Arikan et al., "Urine neutrophil gelatinase-associated lipocalin is an early marker of acute kidney injury in critically ill children: a prospective cohort study," Critical Care, vol. 11, no. 4, article R84, 2007.

[19] S. M. Bagshaw, M. Bennett, M. Haase et al., "Plasma and urine neutrophil gelatinase-associated lipocalin in septic versus nonseptic acute kidney injury in critical illness," Intensive Care Medicine, vol. 36, no. 3, pp. 452-461, 2010.

[20] J. Mårtensson, M. Bell, A. Oldner, S. Xu, P. Venge, and C.-R. Martling, "Neutrophil gelatinase-associated lipocalin in adult septic patients with and without acute kidney injury," Intensive Care Medicine, vol. 36, no. 8, pp. 1333-1340, 2010.

[21] R. P. Dellinger, M. M. Levy, A. Rhodes et al., "Surviving sepsis campaign: international guidelines for management of severe sepsis and septic shock, 2012," Intensive Care Medicine, vol. 39, no. 2, pp. 165-228, 2013.

[22] A. Earley, D. Miskulin, E. J. Lamb, A. S. Levey, and K. Uhlig, "Estimating equations for glomerular filtration rate in the era of creatinine standardization: a systematic review," Annals of Internal Medicine, vol. 156, no. 11, pp. 785-795, 2012.

[23] S. M. Bagshaw, S. Lapinsky, S. Dial et al., "Acute kidney injury in septic shock: clinical outcomes and impact of duration of hypotension prior to initiation of antimicrobial therapy," Intensive Care Medicine, vol. 35, no. 5, pp. 871-881, 2009.

[24] R. L. Mehta, J. A. Kellum, S. V. Shah et al., "Acute Kidney Injury Network: report of an initiative to improve outcomes in acute kidney injury," Critical Care, vol. 11, no. 2, article R31, 2007.

[25] H. Gammelager, C. F. Christiansen, M. B. Johansen, E. Tønnesen, B. Jespersen, and H. T. Sørensen, "Five-year risk of end-stage renal disease among intensive care patients surviving dialysis-requiring acute kidney injury: a nationwide cohort study," Critical Care, vol. 17, no. 4, article R145, 2013.

[26] E. D. Siew, M. E. Matheny, T. A. Ikizler et al., "Commonly used surrogates for baseline renal function affect the classification and prognosis of acute kidney injury," Kidney International, vol. 77, no. 6, pp. 536-542, 2010.

[27] M. Plataki, K. Kashani, J. Cabello-Garza et al., "Predictors of Acute kidney injury in septic shock patients: an observational cohort study," Clinical Journal of the American Society of Nephrology, vol. 6, no. 7, pp. 1744-1751, 2011.

[28] R. Challiner, J. P. Ritchie, C. Fullwood, P. Loughnan, and A. J. Hutchison, "Incidence and consequence of acute kidney injury in unselected emergency admissions to a large acute UK hospital trust," BMC Nephrology, vol. 15, article 84, 2014.

[29] G. R. Stryjewski, E. S. Nylen, M. J. Bell et al., "Interleukin6 , interleukin-8, and a rapid and sensitive assay for calcitonin precursors for the determination of bacterial sepsis in febrile neutropenic children," Pediatric Critical Care Medicine, vol. 6, no. 2, pp. 129-135, 2005.

[30] J. Mishra, M. A. Qing, A. Prada et al., "Identification of neutrophil gelatinase-associated lipocalin as a novel early urinary biomarker for ischemic renal injury," Journal of the American Society of Nephrology, vol. 14, no. 10, pp. 2534-2543, 2003.

[31] J. Mishra, K. Mori, Q. Ma, C. Kelly, J. Barasch, and P. Devarajan, "Neutrophil gelatinase-associated lipocalin: a novel early urinary biomarker for cisplatin nephrotoxicity," American Journal of Nephrology, vol. 24, no. 3, pp. 307-315, 2004.

[32] H. Trachtman, E. Christen, A. Cnaan et al., "Urinary neutrophil gelatinase-associated lipocalcin in D+HUS: a novel marker of renal injury," Pediatric Nephrology, vol. 21, no. 7, pp. 989-994, 2006.

[33] P. Devarajan, "Update on mechanisms of ischemic acute kidney injury," Journal of the American Society of Nephrology, vol. 17, no. 6, pp. 1503-1520, 2006.

[34] L. Cai, J. Borowiec, S. Xu, W. Han, and P. Venge, "Assays of urine levels of HNL/NGAL in patients undergoing cardiac surgery and the impact of antibody configuration on their clinical performances," Clinica Chimica Acta, vol. 403, no. 1-2, pp. 121$125,2009$.

[35] T. L. Nickolas, M. J. O'Rourke, J. Yang et al., "Sensitivity and specificity of a single emergency department measurement of urinary neutrophil gelatinase-associated lipocalin for diagnosing acute kidney injury," Annals of Internal Medicine, vol. 148, no. 11, pp. 810-819, 2008.

[36] S. P. Collins, K. W. Hart, C. J. Lindsell et al., "Elevated urinary neutrophil gelatinase-associated lipocalcin after acute heart failure treatment is associated with worsening renal function and adverse events," European Journal of Heart Failure, vol. 14, no. 9, pp. 1020-1029, 2012. 


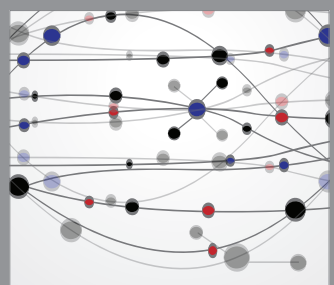

The Scientific World Journal
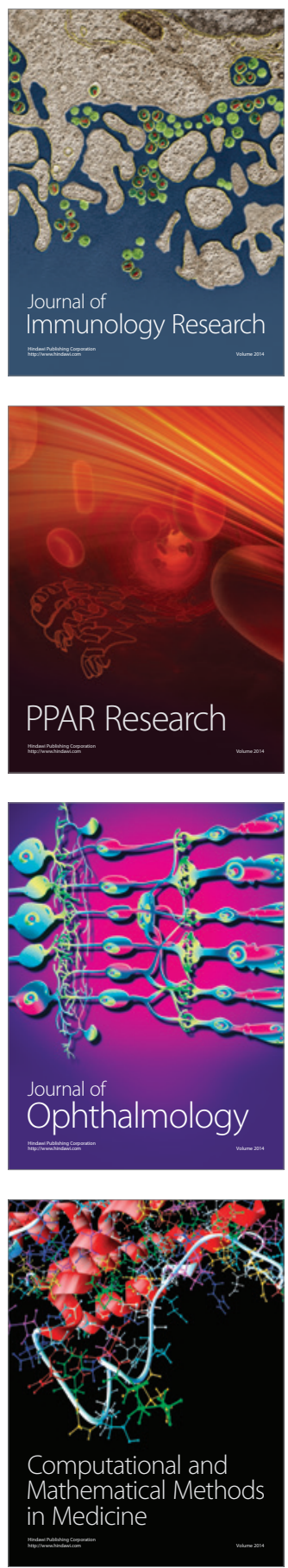

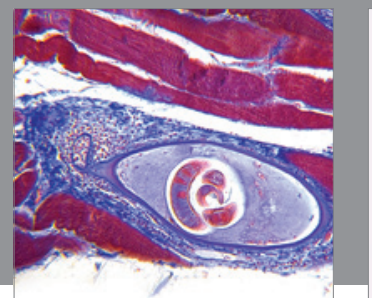

Gastroenterology

Research and Practice
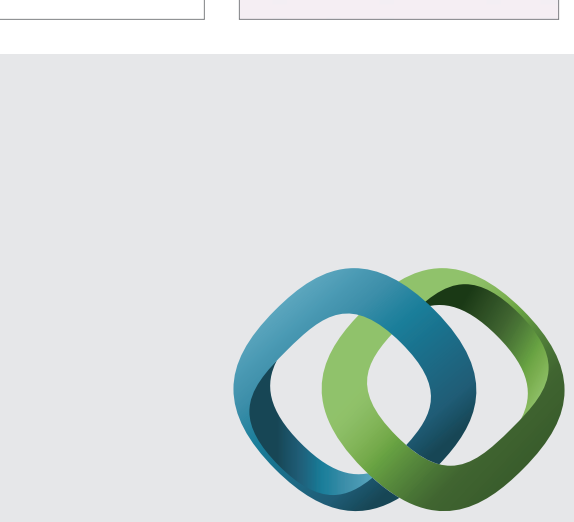

\section{Hindawi}

Submit your manuscripts at

http://www.hindawi.com
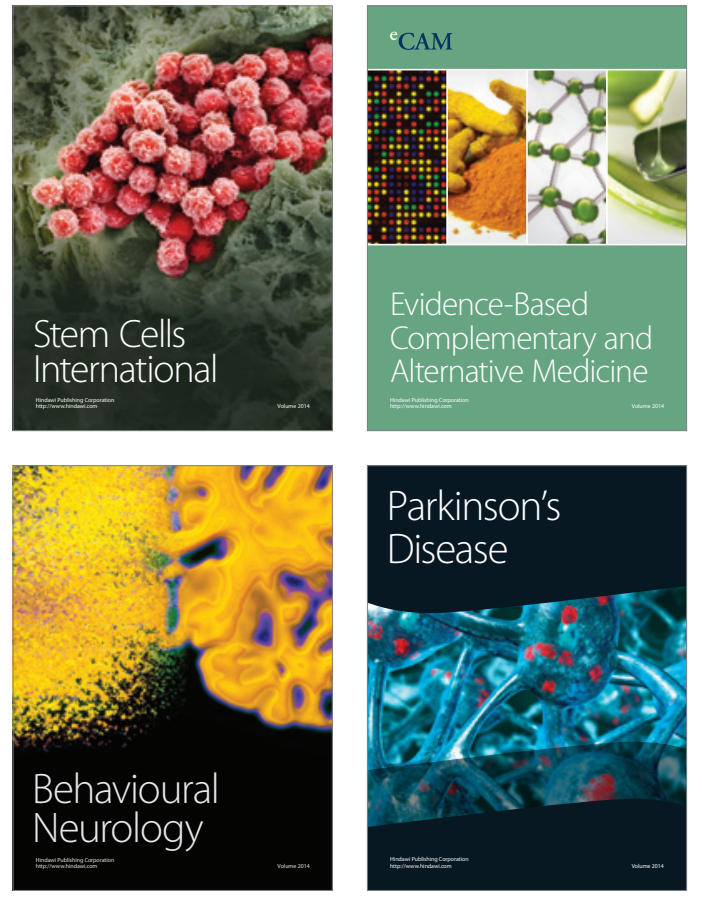
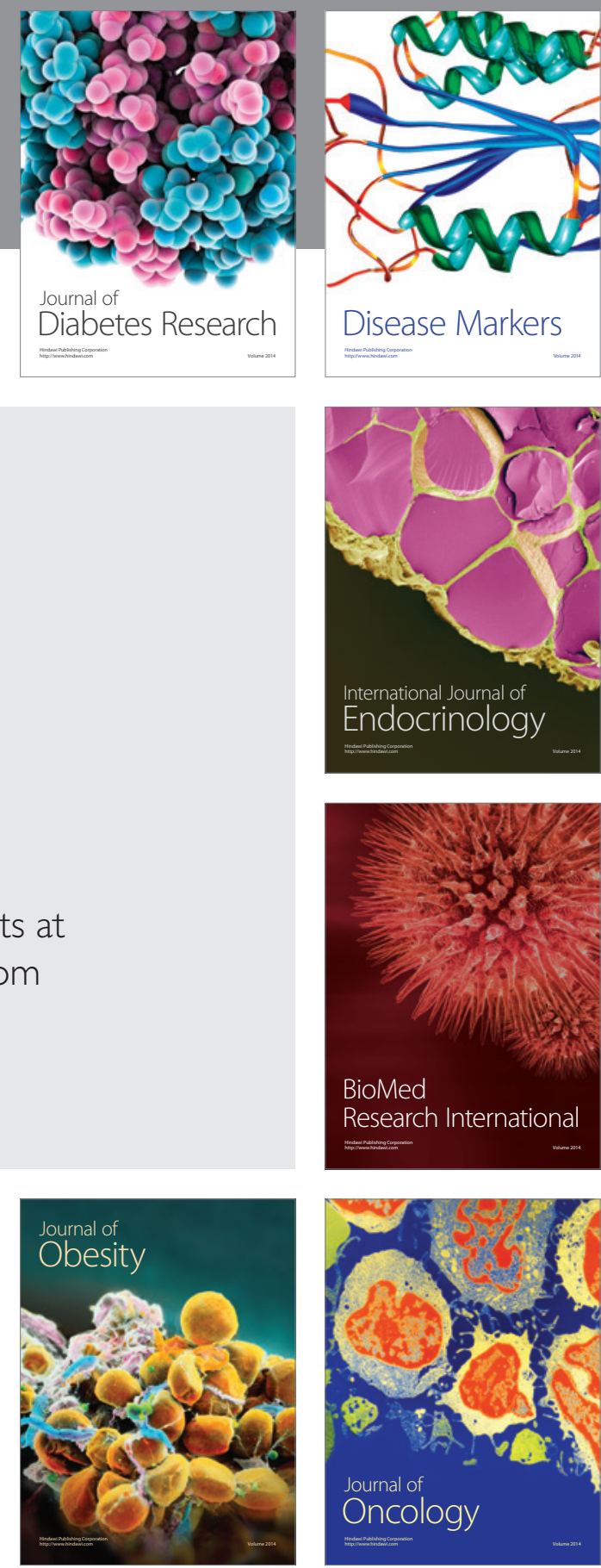

Disease Markers
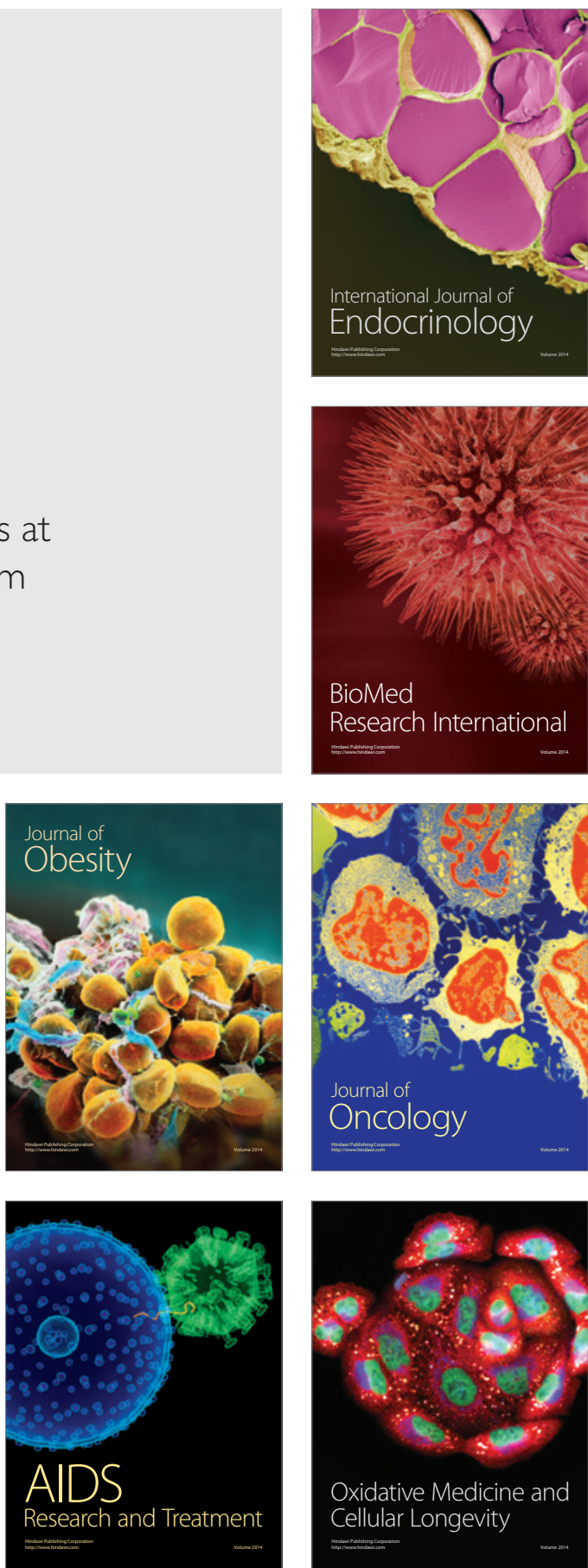